\title{
Towards a European consensus for reporting incidental findings during clinical NGS testing
}

Jayne Y Hehir-Kwa ${ }^{\star}, 1,2$, Mireille Claustres ${ }^{3}$, Ros J Hastings ${ }^{4}$, Conny van Ravenswaaij-Arts ${ }^{5}$, Gabrielle Christenhusz ${ }^{6}$, Maurizio Genuardi ${ }^{7}$, Béla Melegh ${ }^{8}$, Anne Cambon-Thomsen ${ }^{9}$, Philippos Patsalis ${ }^{10}$, Joris Vermeesch ${ }^{11}$, Martina C Cornel ${ }^{12}$, Beverly Searle ${ }^{13}$, Aarno Palotie ${ }^{14}$, Ettore Capoluongo ${ }^{15}$, Borut Peterlin ${ }^{16}$, Xavier Estivill ${ }^{17,18,19}$ and Peter N Robinson ${ }^{20,21,22}$

In 2013, the American College of Medical Genetics (ACMG) examined the issue of incidental findings in whole exome and whole genome sequencing, and introduced recommendations to search for, evaluate and report medically actionable variants in a set of 56 genes. At a debate held during the 2014 European Society for Human Genetics Conference (ESHG) in Milan, Italy, the first author of that paper presented this view in a debate session that did not end with a conclusive vote from the mainly European audience for or against reporting back actionable incidental findings. In this meeting report, we elaborate on the discussions held during a special meeting hosted at the ESHG in 2013 from posing the question 'How to reach a (European) consensus on reporting incidental findings and unclassified variants in diagnostic next generation sequencing'. We ask whether an European consensus exists on the reporting of incidental findings in genome diagnostics, and present a series of key issues that require discussion at both a national and European level in order to develop recommendations for handling incidental findings and unclassified variants in line with the legal and cultural particularities of individual European member states.

European Journal of Human Genetics (2015) 23, 1601-1606; doi:10.1038/ejhg.2015.111; published online 3 June 2015

\section{INTRODUCTION}

O linical next generation sequencing (NGS) is being implemented for the genetic diagnosis of patients with suspected genetic disorders and cancers. ${ }^{1-4}$ These developments have been paired with discussions surrounding the ethics and protocols for reporting incidental findings and unclassified variants (UV). ${ }^{5-11}$ To further develop these concepts the Genetic Services Quality Committee of the European Society of Human Genetics (ESHG) hosted a meeting with invited participants representing laboratory specialists, clinical geneticists, lawyers, ethicists, patient groups and bioinformaticians on June 11th 2013 in Paris. The participants represent a broad range of disciplines as well as European countries including Belgium, Cyprus, Finland, France, Germany, Hungary, Italy, the Netherlands, Slovenia, Spain and UK and were presented with a number of key questions. We report here on the discussion generated from each of these questions, as well as a survey to determine the current level of consensus in reporting genetic laboratory results targeted towards European laboratories currently using NGS technologies in patient care.

Guidelines exist for the classification of variants in a diagnostic setting; ${ }^{12,13}$ however, our knowledge of the genome and techniques for analysing NGS data are still being developed. As a result it is often not possible to definitively classify a variant as being clinically relevant. More recently within the context of NGS, guidelines have been developed for interpreting low frequency variants with a high impact, ${ }^{14}$ which included assessing both positive and negative evidence at the gene and variant level. Furthermore, EuroGenTest (http://www. eurogentest.org) is currently in the process of developing guidelines for diagnostic NGS testing.

Findings generated during clinical NGS testing can be described as:

- Clinically relevant to the diagnostic question

- Clinically or socially relevant for the individual or his/her family members, for example, reproductive relevance to other members, but not relevant to the diagnostic question (incidental findings)

- Not clinically relevant, ('neutral' variants, which are not reported)

- Variants of unknown clinical significance, but potentially related to the primary clinical question (these findings may change status over time).

What is an incidental finding (IF) within the context of clinical NGS sequencing?

The term 'incidental finding' is often applied inconsistently. Uses of the term incidental finding include (i) unexpected positive findings,

${ }^{1}$ Department of Human Genetics, Radboud UMC, Nijmegen, The Netherlands; ${ }^{2}$ Donders Centre of Cognitive Science, Nijmegen, The Netherlands; ${ }^{3}$ Department of Molecular Genetics, IURC (Institut Universitaire de Recherche Clinique), Montpellier, France; ${ }^{4}$ CEQAS, Women's Centre, John Radcliffe Hospital, Oxford University Hospitals NHS Trust, Oxford, England; ${ }^{5}$ University of Groningen, University Medical Center Groningen, Department of Genetics, Groningen, The Netherlands; ${ }^{6}$ Centre for Biomedical Ethics and Law, University of Leuven, Leuven, Belgium; ' Institute of Medical Genetics, 'A. Gemelli' School of Medicine, Catholic University of the Sacred Heart, Rome, Italy; ${ }^{8}$ Department of Medical Genetics, University of Pécs, Pécs, Hungary; ${ }^{9}$ Institut national de la santé et de la recherche médicale and Université Toulouse III-Paul Sabatier joint Unit 1027, Faculty of Medicine, Toulouse, France; ${ }^{10}$ Translational Genetics Team, The Cyprus Institute of Neurology \& Genetics, Cyprus School of Molecular Medicine, Nicosia, Cyprus; ${ }^{11}$ Centre for Human Genetics, University Hospital Leuven, Department of Human Genetics, KU Leuven, Leuven, Belgium; ${ }^{12}$ Clinical Genetics \& EMGO Institute for Health and Care Research, VU University Medical Center, Amsterdam, The Netherlands; ${ }^{13}$ Unique (Rare Chromosome Disorder Support Group), Oxted, England; ${ }^{14}$ Institute for Molecular Medicine Finland (FIMM), University of Helsinki, Helsinki, Finland; ${ }^{15}$ SIBioC Clinical Molecular Diagnostic Training School, Istituto di Biochimica Clinica, Università Cattolica del S. Cuore, Rome, Italy; ${ }^{16}$ Department of Obstetrics and Gynecology, Clinical Institute of Medical Genetics, University Medical Centre Ljubljana, Ljubljana, Slovenia; ${ }^{17}$ Center for Genomic Regulation (CRG), Barcelona, Spain; ${ }^{18}$ Pompeu Fabra University (UPF), Barcelona, Spain; ${ }^{19}$ Dexeus Women's Health Quiron-Dexeus University Institute, Barcelona, Spain; ${ }^{20}$ Institut für Medizinische Genetik und Humangenetik. Charité - Universitätsmedizin Berlin, Berlin, Germany; ${ }^{21}$ Berlin Brandenburg Center for Regenerative Therapies, Charité Universitätsmedizin Berlin, Berlin, Germany; ${ }^{22}$ Department of Mathematics and Computer Science, Institute for Bioinformatics, Freie Universität Berlin, Berlin, Germany *Correspondence: Dr JY Hehir-Kwa, Department of Human Genetics, Radboud UMC, Radboudumc, PO Box 9101, 6500 HB Nijmegen, The Netherlands. Tel: +31 24 3613864; Fax: +31 24 3668752; E-mail: Jayne.Hehir-Kwa@radboudumc.nl

Received 22 August 2014; revised 20 April 2015; accepted 24 April 2015; published online 3 June 2015 
and (ii) the deliberate search for pathogenic variants not related to the primary diagnostic question. ${ }^{15}$ The concept of an incidental finding as an unexpected pathogenic finding, whether or not in retrospect related to the clinical phenotype, is not new to the field of genetics, since any genome-wide technology has the potential to identify incidental findings. ${ }^{16}$ However, due to the large number of variants discovered with whole exome and whole genome sequencing, the probability of discovering an incidental finding increases to an estimated level of $1,{ }^{12}$ $3.4,{ }^{17}$ or even $8.8 \%{ }^{18}$ Differences in these frequencies can be attributed to the ethnicity of individuals, as the vast majority of known pathogenic mutations in databases have been discovered in Caucasian populations, (fewer known pathogenic mutations have been reported in other populations), and the patient selection criteria.

Furthermore as the size of gene panels used for targeted re-sequencing or in silico filtering with exome sequencing increases, so does the chance of detecting an incidental finding. Controversy still remains as to the exact meaning of the term 'incidental finding. ${ }^{19}$ Nonetheless, there is a general plea from patient groups and clinicians that consistent terminology must be used.

A major concern with the term 'incidental finding' is that this implies that the finding was either an incident or not expected, whilst the discovery of such variants is intrinsic to genome-wide screening technologies. Other terms such as 'unexpected' or 'secondary' are equally problematic. Despite the fact that the term 'unsolicited finding' may be better terminology, 'incidental finding' is now commonly used. Therefore it will be employed in this report.

\section{What is an UV or likely pathogenic variant within the context of clinical NGS sequencing?}

Variants for which pathogenicity is unknown can be termed 'unclassified' or, alternatively, 'variants of uncertain significance'. However there is a difference between these two concepts: the term 'unclassified' implies that no attempt has yet been made to classify the variant with regard to its potential pathogenicity, whereas the term 'uncertain significance' intuitively describes that the available evidence for classification is insufficient or contradictory. ${ }^{20,21}$ Similarly variants that seem to be pathogenic, but for which definite proof of pathogenicity is not available, are often denoted with the term 'likely pathogenic'. The classes used to classify variants for which the impact is uncertain also vary and are determined by the use of a 3, 4 or 5 system of variant categories. ${ }^{22}$ Since variants that have not yet been classified are by definition also of uncertain significance, we will use the term UV throughout this report and focus on UV, or variants of uncertain significance that are related to the primary clinical question. As our knowledge of the genome evolves, so will our ability to classify variants. Hence the term UV is time dependent and over time, with accruing knowledge and evidence, a variant may be reclassified to a clinically actionable category.

\section{Do we have an obligation to report and analyse IFs?}

It is important to define the meaning of the term 'analyse' in the context of clinical NGS testing. This includes both the identification, variant calling and in silico interpretation steps. The latter also determines overlap with known disease causing variants but does not necessarily imply proving the biochemical or functional impact of all variants of potential clinical relevance, which is an unrealistic goal in a clinical setting. Without the ability to perform high-throughput functional analysis, or access to relevant tissues, functional testing of all potentially clinically relevant variants remains out of reach for diagnostic laboratories, especially within the context of whole genome sequencing.
Testing laboratories have several options when reporting UVs and IFs, ranging from not reporting them to reporting all UVs/IFs in an appendix of the medical record, hence making use of the existing medical system infrastructure. Often the decision on when and what to report to the patient is given to the clinician. The clinician facilitates the process of reporting IFs, which involves interaction with the patient, for example to taking into account family history, which can further help to predict the consequences of an IF (and over time, also of UVs). Yet this places a large burden on the clinicians to decide what information is appropriate to share. This raises the question of who is the appropriate gate keeper to an individual's genetic information?

Reporting IFs. The ESHG guidelines ${ }^{23}$ state that targeted diagnostic testing should always be performed where possible to minimise the likelihood of detecting IFs. ${ }^{24}$ Even if the technology captures the whole genome, should the analysis be limited to the diagnostic question? Previously, research and diagnostic samples could be clearly distinguished into those for which screening (analysing genomic regions outside the primary clinical question) can and cannot be performed. However, these groups are no longer clearly separated ${ }^{11}$ but according to the American College guidelines ${ }^{12}$ there is no obligation to perform screening on research samples. However, this does not answer the question whether or not we also have an obligation to perform screening when performing diagnostics. Analogies can be made with visiting a general practitioner with a sore throat, but the general practitioner notices a lump on your neck, and refers the patient to the oncologist. Should we apply the same approach to clinical genetic testing?

Given these ethical dilemmas, genetic counselling and informed consent are essential. However this represents a contradiction to the analogy of seeing a general practitioner, as pre-counselling or informed consent are not performed within this context. This in turn raises the question of whether the field of genetics is any different to other medical specialties. Should the medical field of genetics conform, or should other medical specialties adopt current medical genetics practices and perform more extensive informed consent? More recently, the ACMG has issued guidelines on reporting IFs, including an amendment allowing a patient opt-out clause. ${ }^{25}$ In contrast, an ESHG policy statement advises that genetic testing should aim to be targeted at the primary clinical question. ${ }^{6,23}$ At face value these views appear to represent two extremely different points of view. Yet recent discussions during the ESHG 2014 conference in Milan, Italy, and the European Conference on Rare Diseases \& Orphan Products 2014 in Berlin, Germany, suggest that a general consensus may be attained in the near future. With the ACMG suggestion of an opt out for testing IFs, patients are again given the choice of determining the level of testing to be performed, which has been a key issue during many discussions. Furthermore, screening a minimum set of genes for variants is not yet fully implemented in many US diagnostic laboratories. Perhaps the greatest concern raised is the possibility of reporting a false positive IF to a patient and the potential impact this can have. To completely exclude this possibility, half of all respondents to our survey (Table 1) indicated that they do not report any IF. However, other centres do report IFs, but only once the variant has been discussed by a board including an independent doctor, ethicist and lawyer. Clearly based on the results of this small survey at present there is no consensus between laboratories currently performing NGS on reporting IFs.

One important consequence of reporting of IFs in a large number of genes is that laboratories and clinicians will need to develop competency and experience about genes and diseases outside of their 
Question

1. What type of testing is performed on the NGS technology?

2. Do you have a separate informed consent form for your NGS tests? Yes

Whole genome sequencing 2

Whole exome sequencing 6

Large gene panels ( $>20 \quad 7$

genes)

Small gene panels $(<20 \quad 4$

genes)

Yes 7

No 2

3. Are the NGS diagnostic tests performed in your laboratory accredited by an external quality assurance body (eg, ISO, or some Yes country-specific quality accreditation)?

No

4. Are 'incidental findings' reported back to the patient? (ie, variants identified which are classified as pathogenic but do not relate to No the primary clinical question).

5. Do you report 'unclassified variants'? (ie, variants for which the pathogenicity is unclear).

Yes, in a separate report 3

Yes, within the same report 1

Yes 3

No, but they are available upon 2 request

No 4

6. Do you re-analyse your NGS data to determine if variants once classified as UVS can be reclassified?

Yes, initiative of the laboratory 7

No 2

Yes 0

No 9

Yes 8

8. Is NGS testing reimbursed by the health insurance system or government in your country?

No

*Responses were received from laboratories in France, Italy, The Netherlands, Slovenia, Germany and UK.

clinical focus in order to provide accurate and useful interpretations. Indeed this is true of diagnostic exome and genome testing in general. This is an important challenge to the genomics diagnostics community, especially given recent reports of erroneous or incomplete mutation entries in commonly used databases. ${ }^{26,27}$

\section{Who has a duty to disclose IFs to patients?}

It is not the role of the clinical lab to disclose an IF to a patient, but the role of the medical professional requesting the test. The disclosure of such findings requires (additional) detailed information from the patient, and professional counselling, hence should be performed by the responsible clinician. The impact of the IF determines how the finding should be disclosed to a patient. If it has minor consequences or a clinical intervention is possible, then the variant should be reported. Note that this does not relate to low risk variants identified via GWAS studies, as the clinical utility of these variants is yet to be determined. If the variant results in a late onset disorder or has major consequences, counselling and consent will determine if and when the variant can and should be disclosed to the patient. In order to obtain consent, pre-test counselling is needed, and the communication of IFs in combination with post-test counselling is required. This implies that genetic tests should be ordered by medical professionals who are capable of performing sufficient pre-test counselling and that, if this person is not trained in post-test counselling, the patient should be referred when IFs are detected.

The $\mathrm{ACMG}^{12}$ gives guidance on the genes for which IFs should be disclosed to a patient. The advantage of using such a list is it provides a clearly defined subset of genes to investigate, facilitating consistency between different laboratories and aiding automated analysis by bioinformatic pipelines. However, the disadvantage of using a specific list of genes is that it requires frequent updating.
In essence, the reporting of an IF is based on the clinical context, and only clinically actionable IFs should be reported. Clinically actionable variants are defined as the following:

- Provide information which could have an impact on the clinical management of an individual or a family, for example, a BRCA1 mutation.

- Results that are actionable on reproductive grounds, for example, carrier status of a heterozygous CFTR mutation in a child investigated for development delay.

- Are personally actionable, entailing lifestyle change and the reduction of risk factors, for example, familial hypercholesterolaemia ${ }^{28}$ or MCAD deficiency.

Circumstances in which clinically actionable IFs can be reported There is a difference between recording and reporting a variant, as well as between who receives this information and when. Reporting a variant to a clinician does not mean it will be disclosed to the patient. The clinician can evaluate when to convey information based on the clinical history and context. For example, in the case of a familial genetic disorder it is possible that the patient is already aware of a preexisting condition within their family, which was revealed during pretest counselling. The impact of such an IF is therefore different compared to when there is no known family history of the specific disorder associated with the variant. Acute neonatal care is an exceptional context, in which immediate reporting of all IFs to patients' families may not be appropriate. Therefore while reporting the presence of such variants may become appropriate later in life, the clinician may decide not to immediately inform the child's guardians. Other situations in which the reporting of IFs may be postponed 
include when parents or patients are given a diagnosis related to the clinical question that entail a poor prognosis. Likewise the discovery of incidental findings during post-mortem genetic testing may not always be deemed appropriate for immediate reporting. Additional contexts which influence the reporting of incidental findings include, carrier testing, prenatal diagnostics, pharmacogenomics and additional nondiagnostic testing such as medical research (dependant on the study design), forensic testing, parental and genealogical testing. In general, reporting IFs requires good counselling skills and if necessary the patient should be referred for appropriate counselling.

\section{How to manage nondisclosure of socially relevant IFs in reports} Incidents such as misattributed paternity, consanguinity and incest should be reported to the clinician as they may influence subsequent patient management. The clinician will determine whether any further action is required. The only exception to this is incest in cases involving a minor or vulnerable adult, where this must be reported to the country's authorities (by the clinician) dependent on national legislation.

When reporting NGS results the clinical laboratory should consider the extent of the data and information needed to be included in the patient report, as patients have a legal right to view their own medical records as well as the results of a laboratory investigation (dependant on country-specific legislation). For example, a clinical laboratory should have a policy on how non-paternity is reported, this may be by including the level of Mendelian errors in the report but not the interpretation of non-paternity and first verbally reporting the results with the patient's clinician. Formally the laboratory report is part of the medical report. However, usually the medical file and laboratory files are physically or electronically separate entities and if a patient requests to view his or her medical report, the laboratory files are usually not disclosed unless specifically requested.

\section{How much genomic information should be disclosed and to whom?} Medical professionals. Whole exome sequencing and similar tests provide a lot of information about an individual's genome. The disclosure of this information and the timing is case dependent. This includes disclosure to the clinician who has ordered the test, patients (adult $v s$ children) and related family members. For example, the genome sequence of a patient cannot be directly part of the medical report but given as an annex. The referring clinician/geneticist can interrogate the genome sequence when necessary; the findings of this interrogation are then reported in the patient's medical record. Likewise in the near future creating a framework to fully exploit the power of the genome sequencing could result in fewer tests and greater use of the sequencing results. For example by using the secured IT infrastructure of the hospital, a pharmacogenetics specialist may also ask to interrogate the genome, and similarly the results of this test will be reported in the medical record, but not the patient's genome sequence itself. IFs are reported in the medical record when deemed relevant (see criteria for clinically actionable variants).

Non-medical professionals. In addition, many parents and patients believe that it is their right to know and to have their genomic data. This places enormous strains and constraints on genetic testing laboratories for facilitating patient portals to access their sequence data. In fact, none of the laboratories which participated in our survey make sequence data available to patients (Table 1).

Two issues are raised when considering to whom a person's sequence should be accessible:
- The rights and choices of the individual may conflict with those of the family.

- Non-genetic medical professionals having access to genetic information.

In both cases the potential lack of education and knowledge may affect patient management, and therefore the involvement of a genetic counsellor is strongly recommended. Furthermore there are countryspecific legal implications which need to be taken into account.

\section{Parent associations' view on reporting or disclosure of IFs to} patients, to parents of minors and to family members

There is a general plea from patient groups that clinicians use consistent terminology, that tests should be designed to minimise IFs and that genetic testing centres be required to have access to high quality genetic counselling. As part of the counselling process, the family's choice to know or not to know any IF/UV results should ultimately be respected. Whenever possible, results should also be given by a clinical geneticist or genetic counsellor who understands and can explain the test results, using uniform and consistent terminology. Results of uncertain significance must be dealt with carefully and individually. However, in general most parents want to know everything about their child that other people also know. Presenting the data in a clear and unambiguous format is essential. As genetic testing becomes standard medical practice, better genetic and genomic education for non-genetic medical professionals is needed. ${ }^{29}$

\section{Inclusion of quality criteria when reporting variants}

Appropriate quality metrics that provide an assessment of the sensitivity and specificity of the techniques used covering both technical and bioinformatics metrics are needed. For example, the mean coverage, the percentage of target regions above 10x, 20x, 30x coverage, plus the version number and configuration settings of any data analysis and bioinformatics pipeline elements. The metrics should not only document what has been tested but also give the limitations of the test. ${ }^{15}$ Whilst many of these metrics are technology specific and depend on the study design, general guidelines can be given. The criteria should include: (1) how much was sequenced (for example targets covered and depth of sequencing) and how much was not sequenced; (2) The quality of the sequence (such as number of reads with high quality, and the heterozygous/homozygous variant ratio; (3) the method used to identify variants; (4) annotation sources, including but not limited to gene, transcript and variant definitions; (5) data sources used for filtering (such as HGMD, dbSNP); and finally (6) tools and evidence used for assigning pathogenicity to a variant, such as predicting pathogenicity with PolyPhen $2^{\text {(ref. } 30)}$ and MutationTaster. ${ }^{31}$ In all cases, not only the source but also the version of the component should be recorded in such a manner that the bioinformatic pipeline and its results can be reproduced.

\section{Should the testing laboratory store genetic data for future reanalysis?}

The vision that every newborn have their genome sequenced at birth and that their DNA code accompanies them over their lifespan is challenged by practical issues including imperfect sequencing, the massive data storage infrastructure required and cross-border issues created from patient mobility. ${ }^{32}$ Many issues are similar to those associated with electronic medical files although on a much larger scale. Because of the decreasing costs of sequencing and the increasing quality of sequence data, re-sequencing the genome of an individual is a practical alternative if biological material is still available. 
Nonetheless, the results generated from a single sequencing experiment which are used as part of a diagnostic test form part of a medical record. National legislation exists in many individual European member states governing the storage of medical records, but none at the European level.

The storage of data from diagnostic sequencing has two roles; (1) storing the evidence that was used to identify the result(s) of the primary diagnostic question and (2) storage of sequencing results for later reanalysis. The process of identifying mutations in NGS data can broadly be divided into three stages; generation of primary data performed by the sequencer, secondary data which includes derived DNA sequence and alignment of reads, and tertiary interpretation data, including the identification of variants and annotation. Two milestones in data analysis are the primary data (from which all results can be regenerated) and the tertiary interpreted variant files, which can be considered an end product of data analysis and are highly dependent on the steps used during data analysis (for instance, there is a low concordance between several commonly used bioinformatics pipelines for variant calling ${ }^{33}$ ). Even the commonly used BAM file ${ }^{34}$ does not represent primary sequence data, but is the result of aligning sequence reads to a specific reference genome. However, if all sequence reads have been included in the BAM file then it is still possible to regenerate and realign the data. Depending on national legal requirements, as a minimum, the VCF (variant call format) file or equivalent variant file should be stored by the testing laboratory (gVCF. https://sites.google.com/site/gvcftools/home/about-gvcf). But it is recommended that the derived sequence reads are also stored for the length of the diagnosis period (for example FASTQ or BAM files stored for a minimum of two years after sequencing). However as previously stated, no consensus exists within Europe on the length data should be stored from laboratory tests, and a wide variation exists between countries. Storing such a volume of data is a non-trivial task, requiring robust and scalable infrastructure that can guarantee data integrity and allow reanalysis of the data on demand. The sequencing data passes through several stages in its lifespan: (1) working data - the data analysis is on-going; (2) interpretation - data analysis is complete, but the diagnostic report/ interpretation is on-going; (3) active results - the diagnostic report has been made, but the results are being communicated to patients; and (4) archived results - the diagnostic report has been made and communicated, results are archived in case later reanalysis is required.

\section{Should the laboratory re-analyse stored data when interpretation changes/evolves with knowledge?}

There is little consensus as to whether laboratories should re-analyse stored data when interpretation changes and evolves with knowledge. In an ideal world, physicians caring for the patient could trigger a reanalysis of the genomic data if the clinical questions leading to the original analysis remain unanswered and a period of time has gone by such that new findings might identify a clinically relevant variant in the data. However, currently, informatics frameworks that would allow this do not exist, and there has been little discussion surrounding ethical and data privacy issues surrounding data re-analysis. In principle, re-analysis could be implemented by having the laboratory staff re-examine the original sequence data using newer software and databases, or even by allowing patients to check their own data in a Web portal that would allow sequences to be periodically compared with novel findings. Although it is currently unclear how data reanalysis would be funded, most laboratories in our survey are beginning to explore how data reanalysis might be implemented. It does seem to be a consensus that re-analysis should however be limited to the original clinical question, or to additional clinical question(s) requested by the clinician. As laboratories become more automated as a result of IT/bioinformatics developments, it will become simpler to re-analyse stored data. However, the burden of reanalysing NGS data is not only confined to data analysis; reanalysis but also generates a significant work load upon the whole health system through reporting, and re-contacting of patients. Criteria should therefore be determined by genetic centres as to when reanalysis should be undertaken.

\section{CONCLUSIONS AND OUTLOOK}

The widespread implementation of NGS techniques in diagnostic laboratories raises the need for standardised quality of testing and consensus surrounding key issues involving how IF and UVs are reported. Whilst patient care must remain the primary goal within a diagnostic setting, both IFs and UVs play a critical role in expanding our understanding of the complete genotype and phenotype spectrum of a disorder. ${ }^{34,35}$ Hence it is important to develop national and European guidelines for NGS-based diagnostics that include recommendations for the handling of IFs in a way that respects national and European laws and cultural norms.

\section{CONFLICT OF INTEREST}

The authors declare no conflict of interest.

\section{ACKNOWLEDGEMENTS}

We thank all participants of the survey for providing useful insight into how clinical NGS testing has been implemented in their laboratories. Further a special thanks to Wendy Jones for the useful discussions. The research leading to these results has partly received funding from the European Community's Seventh Framework Programme CAGEKID, Cancer genomics of the kidney, $\mathrm{GA} \mathrm{N}^{\circ}$ 241669; ESGI, European Sequencing and Genotyping Infrastructure GA $\mathrm{N}^{\circ}$ 262055; GEUVADIS, Sharing capacity across Europe in high-throughput sequencing technology to explore genetic variation in health and disease, $\mathrm{GA} \mathrm{N}^{\circ}$ 261123; 3 Gb-TEST, Introducing diagnostic applications of ' 3 Gb-testing' in human genetics, GA $N^{\circ}$ 602269). PNR is supported by a grant from the Bundesministerium für Bildung und Forschung (BMBF project number 0313911).

1 de Ligt J, Willemsen MH, van Bon BW et al: Diagnostic exome sequencing in persons with severe intellectual disability. N Engl J Medicine 2012; 367: 1921-1929.

2 Rauch A, Wieczorek D, Graf E et al: Range of genetic mutations associated with severe non-syndromic sporadic intellectual disability: an exome sequencing study. Lancet 2012; 380: 1674-1682.

3 Yang Y, Muzny DM, Reid JG et al: Clinical whole-exome sequencing for the diagnosis of mendelian disorders. The N Engl J Medicine 2013; 369: 1502-1511.

4 Zemojtel T, Kohler S, Mackenroth L et al: Effective diagnosis of genetic disease by computational phenotype analysis of the disease-associated genome. Sci Tans/ Med 2014; 6: 252ra123.

5 McGuire AL, Joffe S, Koenig BA et al: Point-counterpoint. Ethics and genomic incidental findings. Science (New York, NY) 2013; 340: 1047-1048.

6 vanEI CG, Dondorp WJ: de Wert GM, Cornel MC: call for prudence in whole-genome testing. Science (New York, NY) 2013; 341: 958-959.

7 Wolf SM, Annas GJ, Elias S: Point-counterpoint. Patient autonomy and incidental findings in clinical genomics. Science (New York, NY) 2013; 340: 1049-1050.

8 Burke W, Matheny Antommaria AH, Bennett R et al: Recommendations for returning genomic incidental findings? We need to talk! Genet Med 2013; 15: 854-859.

9 Berg JS, Amendola LM, Eng $C$ et al: Processes and preliminary outputs for identification of actionable genes as incidental findings in genomic sequence data in the Clinical Sequencing Exploratory Research Consortium. Genet Med 2013; 15: 860-867.

10 Anderson JA, Hayeems R, Shuman C et al: Predictive Genetic Testing for Adult-Onset Disorders in Minors: A Critical Analysis of the Arguments For and Against the 2013 ACMG guidelines. Clin Genet 2014; 87: 301-310.

11 Cambon-Thomsen A: [Actors and tools of predictive genetics: ethics at the heart of governance] Acteurs et outils de la prédiction génétique: l'éthique au cœur de la gouvernance. Journal international de bioéthique et d'éthique des sciences 2014; 25 : 165-174. 
1606

12 Rehm HL, Bale SJ, Bayrak-Toydemir P et al: ACMG clinical laboratory standards for next-generation sequencing. Genet Med 2013; 15: 733-747.

13 Wallis Y, Payne S, McAnulty C et al: Practice Guidelines for the Evaluation of Pathogenicity and the Reporting of Sequence Variants in Clinical Molecular Genetics 2013.

14 MacArthur DG, Manolio TA, Dimmock DP et al: Guidelines for investigating causality of sequence variants in human disease. Nature 2014; 508: 469-476.

15 Allyse M, Michie M: Not-so-incidental findings: the ACMG recommendations on the reporting of incidental findings in clinical whole genome and whole exome sequencing. Trends Biotechnol 2013; 31: 439-441.

16 Anastasova V, Blasimme A, Julia S, Cambon-Thomsen A: Genomic incidental findings: reducing the burden to be fair. The Am Journal of bioeth 2013; 13: 52-54.

17 Dorschner MO, Amendola LM, Turner EH et al: Actionable, pathogenic incidental findings in 1,000 participants' exomes. Am J Hum Genet 2013; 93: 631-640.

18 Lawrence L, Sincan M, Markello T et al: The implications of familial incidental findings from exome sequencing: the NIH Undiagnosed Diseases Program experience. Genet Med 2014; 16: 741-750.

19 Anastasova V, Mahalatchimy A, Rial-Sebbag E et al: Communication of results and disclosure of incidental findings in longitudinal paediatric research. Pediatr allergy and immunology: official publication of the European Society of Pediatr Allergy Immunol 2013; 24: 389-394.

20 Plon SE, Eccles DM, Easton D et al: Sequence variant classification and reporting: recommendations for improving the interpretation of cancer susceptibility genetic test results. Human Mutation 2008; 29: 1282-1291.

21 Thompson BA, Spurdle AB, Plazzer JP et al: Application of a 5-tiered scheme for standardized classification of 2,360 unique mismatch repair gene variants in the InSiGHT locus-specific database. Nat Genet 2014; 46: 107-115.

22 Wallis $\mathrm{Y}$, Payne S, McAnulty $\mathrm{C}$ et al: Practice Guidelines for the Evaluation of Pathogenicity and the Reporting of Sequence Variants in Clinical Molecular Genetics. UK Clinical Molecular Genetics Society (ACGS) \& Dutch Society of Clinical Genetic Laboratory Specialists (VKGL) 2013.
23 Claustres M, Kozich V, Dequeker E et al: Recommendations for reporting results of diagnostic genetic testing (biochemical, cytogenetic and molecular genetic). Eur J Hum Genet 2013; 22: 160-170.

24 van El CG, Cornel MC, Borry P et al: Whole-genome sequencing in health care: recommendations of the European Society of Human Genetics. Eur J Hum Genet 2013; 21: 580-584.

25 American College of Medical Genetics and Genomics: Incidental findings in clinical genomics: a clarification. Genet Med 2013; 15: 664-666.

26 Gout AM, Ravine D: AGV Consortium Analysis of published PKD1 gene sequence variants. Nat Genet 2007; 39: 427-428.

27 Bell CJ, Dinwiddie DL, Miller NA et al: Carrier testing for severe childhood recessive diseases by next-generation sequencing. Sci Transl Med 2011; 3: 65 ra64.

28 Robinson JG: Management of familial hypercholesterolemia: a review of the recommendations from the National Lipid Association Expert Panel on Familial Hypercholesterolemia. J Manag Care Pharm 2013; 19: 139-149.

29 Middleton A, Parker M, Wright CF, Bragin E, Hurles ME: Empirical research on the ethics of genomic research. Am J Med Genet A 2013; 161A: 2099-2101.

30 Adzhubei IA, Schmidt S, Peshkin L et al: A method and server for predicting damaging missense mutations. Nat Methods 2010; 7: 248-249.

31 Schwarz JM, Cooper DN, Schuelke M, Seelow D: MutationTaster2: mutation prediction for the deep-sequencing age. Nat Methods 2014; 11: 361-362.

32 Capps B, Chadwick R, Chalmers DRC Imagined futures: capturing the benefits of genome sequencing for society 2013.

33 O'Rawe J, Jiang T, Sun G et al: Low concordance of multiple variant-calling pipelines: practical implications for exome and genome sequencing. Genome Med 2013; 5: 28.

$34 \mathrm{Li} \mathrm{H}$, Handsaker B, Wysoker A et al: The Sequence Alignment/Map format and SAMtools. Bioinformatics (Oxford, England) 2009; 25: 2078-2079.

35 Biesecker Leslie G: Incidental Variants Are Critical for Genomics. Am J Hum Genet 2013; 92: 648-651. 